S. Bergeret MD, I.H. Gaudy MD, J.F. Boitier MD, F. Ferracci MD

\title{
Rôle de l'acidose métabolique dans la tachypnée provoquée par l'alfatésine chez le chien
}

\begin{abstract}
Chez le chien anesthésiê avec de l'Alfatésine, en cas d'anesthésie légère, on note uné tachypnée. Il existe également une acidose métabolique qui peut jouer un rôle dans l'augmeniation de la fréquence respiratoire. Les effets ventilatoires de la correction de l'acidose métabolique ont été étudiès chez cing chiens anesthésiés avec de l'Alfatésine adminisirée à débit constant $6.06 \pm 2.67$ $\mu l \cdot \mathrm{kg}^{-1} \cdot \mathrm{min}^{-1}$ ). Le régime ventilatoire (durée de l'inspiration, Ti; durée de l'expiration, TE; durée de la période respiratoire, Ttor; fréquence, $f$; rapport Ti(Tiot), le volume courant $(V \mathrm{~T})$, la ventilation minute (VE), le débit ventilatoire moyen ( $V T / T)$, et les gaz du sang, $p H a$, $\mathrm{PaCO}_{2}, \mathrm{PaO}_{2}$, ont été mesurés avant et après l'administration de $2.94 \pm 1.46 \mathrm{ml} \cdot \mathrm{kg}^{-i}$ de bicarbonate de sodium à 42 pour mille $\left(495 \mathrm{mmol} \cdot \mathrm{l}^{-1}\right)$. Le pH artériel est passé de $7.27 \pm 0.10$ à $7.44 \pm 0.20(p<0.05)$. $/$ n n $^{\prime} y$ a pas eu de variation significative des autres paramètres, en particulier de la fréquence respiratoire qui est passée de $41 \pm 10.5$ à $43.3 \pm 17.2$ par minute. L'acidose métabolique n'explique donc pas la tachypnée. Les mécanismes possibles de la tachypnée, en particulier le rôle de l'histamine, sont discutés.
\end{abstract}

\section{Key words}

ANAESTHETICS, INTRAVENOUS: Althesin, respiratory effects.

Laboratoire de Recherche Anesthésiologique Hôpital Rothschild, 33 Bvd de Picpus, 75012, Paris.

Demandes de tirés à part: Professeur J.H. Gaudy

Service d'Anesthésie-Réanimation, Hôpital Rothschild 33, Bvd de Picpus, 75012, Paris.

Ce travail a été rćalisé grace à l'aide de l'Amicale des Anesthésistes de l'Hôpital Rothschild et de la Faculté de Médecine Saint-Antoine.
Les effets des anesthésiques gênéraux sur le régime ventilatoire ont été très étudiés. La plupart des anesthésiques provoquent une tachypnée, que ce soient les anesthésiques par inhalation ${ }^{1}$ ou les anesthésiques intraveineux. ${ }^{2}$ Chez l'homme, une tachypnée est fréquemment observée lors de l'injection intraveineuse d'Alfatésine. ${ }^{3-5}$ Récemment, dans ce journal, Gaudy at al. ${ }^{6}$ ont rapporté chez le chien qu'en cas d'anesthésie légère il existe une. tachypnée. Ces auteurs ont attribué cette tachypnée à une action centrale de l'anesthésique. L'analyse de leurs résultats montre qu'il existe une acidose métabolique ( $\mathrm{pHa}=7.28 \pm 0.04 ; \mathrm{PaCO}_{2}=4.60 \pm$ $0.57 \mathrm{kPa}$ ). L'acidose observée pourrait expliquer en partie l'augmentation de la fréquence respiratoire. Le présent travail se propose d'étudier dans les mêmes conditions expérimentales que celles de Gaudy et collaborateurs les effets ventilatoires de la correction de l'acidose métabolique chez le chien en anesthésie légère, sous Alfatésine.

\section{Méthodes}

L'étude a été réalisée chez cinq chiens, mâles, de race beagle (poids, moyenne $\pm \mathrm{SD}=17.2 \pm$ $5.7 \mathrm{~kg}$ ), à jeûn depuis 12 heures, non prémédiqués). Une veine d'un membre antérieur a été cathétérisée (gauge 21) et l' anesthésie a été induite puis conduite avec de l'Alfatésine diluée dans une solution salée isotonique, administrée à l'aide d'une pompe électrique (Rhone Poulenc RP 04 PE). Après induction de l'anesthésie, un tube trachéal à ballonnet (Portex $N^{\circ} 8.5$ ) a été mis en place et le ballonnet a été gonflé de manière à assurer une étanchéité parfaite. Les animaux respirant à l'air ambiant ont été placés en décubitus latéral gauche, sur un matelas chauffant thermostaté, la température rectale étant maintenue à $37^{\circ} \mathrm{C}$. Une autre veine a été cathétérisée afin 
d'administrer éventuellement un soluté de remplissage vasculaire (Ringer lactate). Un catheter a été introduit par voic percutanée dans une artère fémorale afin de mesurer la pression artérielle (capteur Statham P23 IA) et de recueillir des échantillons de sang artériel pour la mesure de $\mathrm{pHa}, \mathrm{PaCO}_{2}$ et $\mathrm{PaO}_{2}$; les mesures ont été effectuées dans les cinq minutes suivant le prélèvement (Instrument Laboratories 313). La concentration de gaz carbonique dans les gaz expirés (FCO2) a été surveillée en continu (Analyseur Beckman LB2). Le spirogramme a été obtenu après intégration du signal pneumotachographique (Fleisch $\mathrm{N}^{\mathrm{v}} 2$ ). La pression artérielle systémique, $\mathrm{FCO}_{2}$, et le spirogramme (débit, $\dot{V}$ et volume courant, VT) ont été enregistrés sur un polygraphe (Beckman R 411) à la vitesse de $25 \mathrm{~mm} / \mathrm{sec}$. La durée de l'inspiration, $T r$, la durée de l'expiration, TE, la période, Ttot ont été mesurées. La fréquence respiratoire TuTtot, la ventilation minute, $V_{T} \times f$ et le débit inspiratoire moyen, $V T / T ı$ ont été calculés.

Après induction de l'anesthésie, le débit d'Alfatésine a été ajustế de manière à obtenir une anesthésie légère, la profondeur de l'anesthésie étant jugée cliniquement. Chaque expérience a débuté après 60 minutes d'état stable jugé sur $\mathrm{FCO}_{2}$, la pression artérielle, la fréquence cardiaque, la fréquence respiratoire, le volume courant et la température. Les gaz du sang ont été mesurés et le spirogramme a été enregistré, puis $2.94 \pm 1.46 \mathrm{ml}$. $\mathrm{kg}^{-1} \mathrm{de}$ bicarbonate à 42 pour mille $\left(495 \mathrm{mmol} \cdot \mathrm{l}^{-1}\right)$ ont été perfusés en 10 minutes. Cinq minutes plus tard, le spirogramme et les gaz du sang ont êté à nouveau meșurés. Pour le traitement statistique des résultats, le test $t$ de Student sur des séries appariées a été utilisé.

\section{Résultats (Tableau)}

Les effets cliniques observés dans la présente étude sont identiques à ceux rapportés dans d'autres études. ${ }^{6-9}$ L'induction de l'anesthésie s'est accompagnée d'un rash cutané généralisé, d'un oedème du museau, des pattes et des oreilles, ainsi que d'une diminution modérée de la pression artérielle. L'hypotension artérielle a été facilement corrigée par la perfusion de 150 à $200 \mathrm{ml}$ de solution de Ringer lactate. Ensuite la pression artérielle est restée stable. Les débits d'Alfatésine nécessaires pour obtenir une anesthésie légère (avec conservation des réflexes oculopalpébral, oculo-moteur, de déglutition), ont été très variables d'un animal à l'autre (débit moyen $\pm \mathrm{SD}=6.06 \pm 2.67 \mu \mathrm{l} \cdot \mathrm{kg}^{-1}$. $\left.\min ^{-1}\right)$.

Si on compare les résultats observés dans la présente étude aux valeurs normales relevées par Green $^{10}$ et par Stahl ${ }^{11}$ chez des chiens de même poids, on constate que l'anesthésie a provoqué les modifications suivantes: $\dot{V}_{E}$ est resté à la limite supérieure de la normale, la fréquence respiratoire a nettement augmenté, $V_{T}$ est restí à la limite inférieure de la normale, $\mathrm{pHa}$ a diminué alors que $\mathrm{PaCO}_{2}$ est restée normale.

La correction de l'acidose métábolique, pHa passant de $7.27 \pm 0.10$ à $7.44 \pm 0.20$, n'a entrainé aucune modification des paramètres mesurés ou calculés. En particulier, il n'y a pas eu de modification de la fréquence respiratoire $(41 \pm 10.5$ par minute avant l'administration de bicarbonate, 43.3 \pm 17.2 après l'administration de bicarbonate).

\section{Discussion}

La tachypnéc est un phénomènc couramment observé après administration d'agents anesthésiques par inhalation ${ }^{1}$ ou intraveineux, ${ }^{2}$ comme l'Alfatésine. ${ }^{2,6}$ Dans le cas de l'Alfatésine, shez le chien, Gaudy et al. ${ }^{6}$ ont avancé l'hypothèse d'une action centrale stimulante. Les animaux de cette étude présentaient une acidose métabolique susceptible d'expliquer, du moins en partie, la tachypnée.

Dans la présente étude il existe aussi une acidose métabolique. Elle cst plus importante que dans l'étude de Gaudy et al. ( $\mathrm{pHa}=7.27 \pm 0.10$ contre $7.28 \pm 0.04)$. Il est difficile de trouver une explication à cette différence. En effet, dans les deux études, il s'agissait d'animaux de même race, à jeûn depuis douze heures, anesthiésiés avec des doses voisines d'Alfatésine. En l'absence de mesure du pH avant l'anesthesie il est difficile de mesurer le rôle exact de cette demière. L'acidose métabolique observée dans les deux études peut ềre due en partie au jeûne. Cependant, en raison du degré d'acidose observé, en particulier chez certains animaux de la présente étude, il est plus probable que l'acidose est secondaire à la réaction anaphylactoide constatée dans tous les cas et à la phase d'hypotension qui l'accompagne.

Les résultats de la présente étude permettent de conclure que l'acidose métabolique n'est pas responsable de la tachypnée observée chez le chien à la suite de l'administration d'Alfatésine, puisque la 


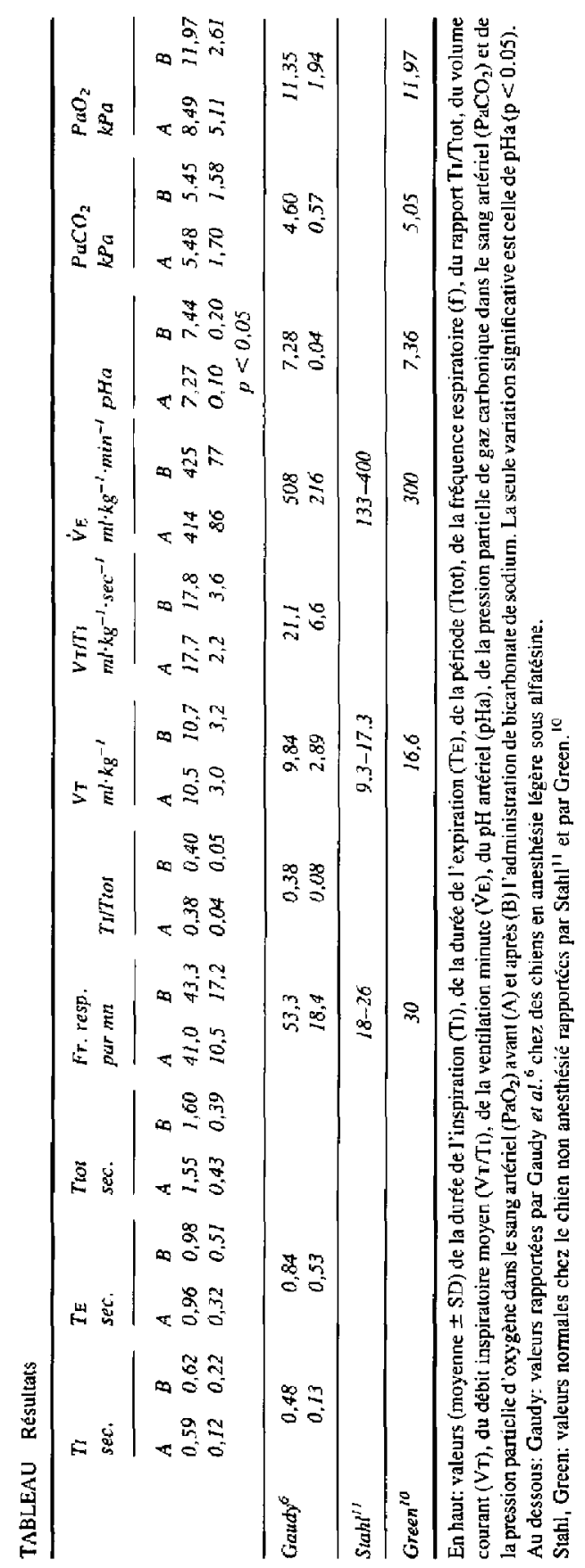


correction de l'acidose métabolique par l'administration de bicarbonate de sodium ne modifie pas la fréquence respiratoire. La tachypnée pourrait être due à la liberation d'histamine par le solvant du mélange alphaxolone-alphadolone, le crémophor EL. Le crémophor EL est un puissant histaminolibérateur chez le chien, ${ }^{9,12}$ libération qui explique les manifestations cliniques observécs chez cet animal: hypotension artérielle, rash cutané, cudème du museau, des oreilles et des pattes, fréquemment rapportées. ${ }^{6-12}$ Le tableau clinique observé dans la présente ćtude, hypotension artérielle modérée et rash cutané, évoque une vasodilatation artérielle périphérique telle qu'elle peut être provaquée par l'injection de faibles doses d'histamine chez le chien. ${ }^{13}$ Cette liberation d'histarnine pourrait expliquer la tachypnée observée. En effet, chez le chat, l'histamine injectée dans le coeur droit stimule les récepteurs J soit directement, ${ }^{14}$ soit plus probablement à la suite des mouvements liquidiens secondaires à l'augmentation de la perméabilité capillaire et a l'augmentation des résistances vasculaires pulmonaires. ${ }^{15}$ Cette stimulation des récepteurs $J$, observée chez le chat après injection de phényldiguanide, s'accompagne d'une tachypnée avec augmentation de la ventilation et hypocapnie. ${ }^{16}$ Mais i] est possible que le chien réponde différemment. En effet, chez cet animal, le seuil de réponse des récepteurs $\mathrm{J}$ est beaucoup plus élevế que chez le chat, ${ }^{17}$ et la libération d'histamine ne $s^{7}$ accompagne pas obligatoirement d'une tachypnée. ${ }^{9}$

Si le rôle de l'histamine ne peut être écarté pour expliquer la tachypnée provoquée par l'Alfatésine chez te chien, une action centrale de l'anesthésique doit être envisagée. En effet, chez le chien, l'augmentation des doses d'Alfatésine provoque une diminution de la fréquence respiratoire, ${ }^{6}$ et les effets ventilatoires de doses croissantes de thiopental, toujours chez le chien, alors qu'il n'y a pas de réaction anaphylactoide, sont comparables à ceux de doses croissantes d'Alfatésine: ${ }^{18}$ la tachypnée observée en cas de faibles doses fait place à une bradypnée en cas de fortes doses alors qu'il n'y a pas de variation de la mécanique respiratoire.

\section{Références}

1 Severinghaus JW, Larson CP Jr. Respiration in anesthesia. In: Handbook of Physiolagy, Respiration, II. Eds. W.O. Fenn et H. Rahn. American Physiological Society, Washington, 1965, pp. 121964.

2 Gautier $H$, Gaudy $J H$. Changes in ventilatory pattern induced by intravenous anesthetic agents in human subjects. J Appl Physiol 1978; 45: 171-6.

3 Clarke RS, Dundee JW, Carson IW, Arora MV, Mc Caughey W. Clinical studies of induction agents. $\mathrm{XL}$ : Althesin with various premedicants. Br J Anaesth 1972; 44: 845-8.

4 Savege TM, Rass L, Foley EI, Maxwell MPA. A comparison of the cardiorespiratory cffects during induction of anaesthesia of althesin with thiopentone and methohexicone. Post Med J 1972; 48, Suppl 2: $66-72$.

5 Whitwam $\sqrt{ } G$. Adverse reactions to I.V. induction agents. Br J Anaesth 1978; 50: 677-87.

6 Gaudy JH, Dauthier C, Boitier JF. Ferracci F Effets ventilatoires de débits croissarits d'alfatésine chez le chien. Can Anaesth Soc J 1982; 29: 600-11.

7 Gaudy JH, Dauthier C, Boitier JF, Ferracci $F$. Réponse ventilatoire à l'hypercapnie et à l'hypoxie hypocapnique du chien anesthésie sous différents niveaux d'anesthésie à l'alfatésine. Ann Fr Anesth Réanim 1982; 1: 395-400.

8 Child KJ, Currie JP, Davis B, Doda's MG, Pearce $D R, T w i s s e l D J$. The pharmacological properties in animals of CT 1341, a new steroid anaesthetic agent. Br J Anaesth 1971; 43: 2-13

9 Lorenz W, Schmal A, Schult $H$ et al. Histamine release and hypotensive reactions in dogs by solubilizing agents and fatty acids: Analysis of various components in cremophor EL and development of a compound with reduced toxicity. Drugs and Actions 1982; 12: 64-80.

10 Green $C J$. Animal Anaesthesia 1st ed. London. Laboratories Animals. Ltd. pp. 207 (1979).

11 Stahl WR. Scaling of respiratory variables in mammals. J Appl Physiol 1967; 22, 453--60.

12 Lorenz W, Reiman HJ, Schmal A et al. Histamine release in dogs by cremophor $\mathrm{EL}$ and its derivatives: oxethylated olcic acid is the most cffective constituent. Agents and Actions 1977; 7: 63-7.

13 Dale HH, Laidlaw PP. Histamine shock. J Physiology $1919 ; 52: 355-90$. 
14 Armstrong DJ, Luck JC. A comparative study of irritant and type $J$ receptors in the cat. Resp Physiol 1974: 21: 47-60.

15 Paintal AS. Mechanism of stimulation of type J pulmonary receptors. J Physiol 1969; 203, 5I1- 32 .

16 Anand A, Loeschke HH, Marek W, Paintal AS. Significance of the respiratory drive by impulse from $J$ pulmonaty receptors. J Physiol 1981; 325: 14

17 Paintal AS. Reflex effects of J receptors. $m$; Central nervous environment. Ed by M.E. Schlafke, H.P. Koepchen and W.R. See. Springer-Verlag, Berlin, Heidelberg 1983; 134-41.

18 Gaudy, JH, Dauthier C, Gallior M, Ferracci $F$, $B o i t i e r J F$. Ventilatory effects produced ty the I.V. administration of incremental doses of thiopentone in the dog. Br J Anaesth 1983; 55:977-84.

\section{Abstract}

In the dog anaesthetized with Althesin, tachypnea has been observed under light anaesthesia. There was also a metabolic acidosis which might be responsible for the increase of the respiratory rate. The ventilatory effects of the correction of the metabolic acidosis were studied in five dogs anaesthetized with Athesin administered at a constant rate of infusion $\left(6.06 \pm 2.67 \mu \mathrm{l} \cdot \mathrm{kg}^{-1} \cdot \mathrm{min}^{-1}\right)$. The ventilatory pattern (duration of inspiration. $T l$; duration of expiration, TE; duration of the respiratory cycle, Ttot; respiratory rate, f; ratio TI/Ttot, tidal volume $(V T)$, minute ventilation $(\dot{V} E)$, mean inspiratory flow $(\mathrm{V} / \mathrm{T} / \mathrm{T})$ and blood gases, $\mathrm{pHa}, \mathrm{PaCO}_{2}, \mathrm{PaO}_{2}$, were measured before and after administration of $42 \mathrm{p}$ mille sodium bicarbonate $\left(495 \mathrm{mmol} \cdot \mathrm{l}^{-1}\right)$. Arterial $\mathrm{pH}$ increased from $7.27 \pm 0.10$ to $7.44 \pm 0.20(p<0.05)$. There was no statistically significam change of other values, particularly for respiratory rate which varied from $41 \pm 10.5$ to $43.3 \pm 17.2$ per minute. Metabolic acidosis does not explain the tachyprea. Mechanisms of tachypnea, particularly the role of histamine, are dis. cussed. 